\title{
Experts call for smarter approach to targeted therapies
}

The current generation of rational cancer drugs promises to usher in a new era by targeting distinct genetic features of tumors. But the paradigm shift in treatment needs to be accompanied by a corresponding boost in diagnostics, experts say.

Cancer drugs have traditionally been evaluated on the basis of their effects on tumor size and patient survival. But researchers say those endpoints are too imprecise for testing targeted drugs.

"We need early returns and we need exit polling," says Todd Golub, associate professor of pediatrics at Harvard University. "If you're using a kinase inhibitor, did you inhibit the kinase? If you didn't, you have no business waiting around to see whether the tumor shrinks."

By their very nature, most targeted drugs have a low response rate. The drug Iressa, for example, dramatically improves survival in lung cancer-but in just $10 \%$ of patients. In April, a year after the drug's approval in the US, two groups independently found that its effect is dependent on a genetic mutation (Science 304, 1497-1500; 2004 and N. Engl. J. Med. 350, 2129-2139; 2004). "The lesson from Iressa is that you have to first determine who is responding," says Brian Druker, chair of leukemia research at Oregon Health \& Sciences University.

Because the drugs are often developed empirically, researchers may not know enough about the mechanism they target to develop diagnostics from the start. A few tests are beginning to emerge, says Charles Sawyers, professor of medicine at the University of California in Los Angeles, but they are still on the level of "Mom-and-Pop molecular assays" developed in small labs, he says. "That kind of stuff has to become mainstream."

If querying tumor tissue for molecular markers is to become routine, collection and storage of biopsy samples will also require standardization. Most centers fix biopsy tissue in formaldehyde or embed it in paraffin, which degrades messenger RNA. Freezing tissue, another common storage technique, disrupts proteins. Researchers will need to either find noninvasive ways to take fresh biopsies, or develop assays that do not require tumor tissue, says George Demetri, director of the Center for Sarcoma and Bone Oncology at the Dana Farber Cancer Institute in Boston.

In the absence of standardized tissue-based assays, some researchers have turned to in vivo molecular imaging. Those methods do not reveal a drug's mechanism of action-and so cannot be used for predictive profiling-but they can detect a tumor's response to treatment within days rather than months.

Developing standardized diagnostics for research and clinical tests will take time, particularly as many of the assays are new. "Even now, the [US Food and Drug Administration] looks at our [imaging] data and says, we don't know what that means," says Demetri. "It can take years to develop that validated data set. You want to be careful that you don't move it forward so fast that you get sloppy."

Alla Katsnelson, New York

\section{Ovarian transplants restore fertility in cancer patients}

Conventional cancer treatments such as chemotherapy and radiation often carry one lasting side effect: sterility. But recent advances offer some hope in preserving the fertility of young female cancer patients.

Belgian researchers reported in June that, for the first time, a woman had become pregnant after receiving transplanted ovarian tissue. The results were presented at the annual meeting of the European Society for Human Reproduction and Embryology.

"If this was indeed a pregnancy that resulted from frozen grafted tissue, then it is an amazing accomplishment," says David Lee, assistant professor of obstetrics and gynecology at the Oregon Health \& Science University in Portland. "It is the ultimate proof of principle - this validates what many people have been working on for many years.'

Many in vitro fertilization clinics routinely freeze embryos. In the US, about ten clinics also freeze the oocytes or eggs. Worldwide, nearly 200 pregnancies have resulted from frozen eggs.

Freezing ovarian tissue offers one alternative that could be used any time a medical treatment might harm ovaries, says Kutluk Oktay, associate professor of obstetrics and

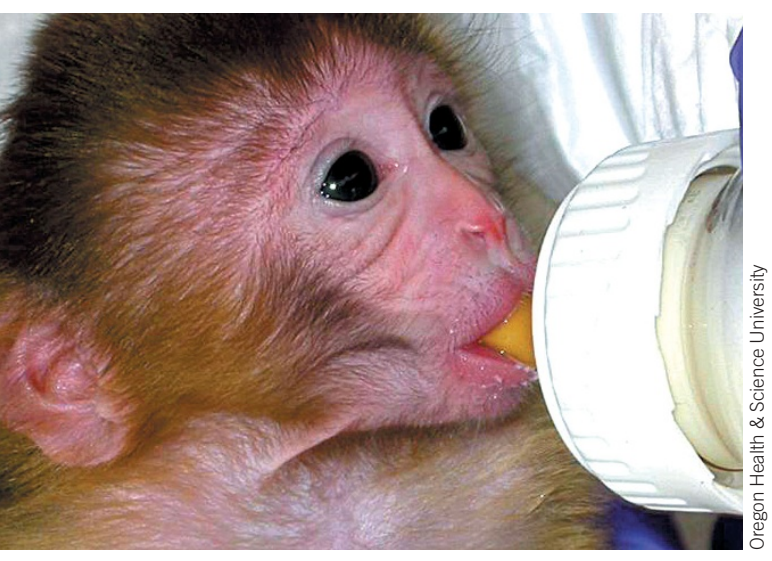

Infant monkey BRENDA was born from transplanted ovarian tissue.

cess with a similar technique in monkeys. To simulate the loss of fertility and the changes in hormone production in women battling cancer, the researchers removed the ovaries of seven anesthetized female monkeys.

They transplanted some of each monkey's own ovarian tissue back into its body and monitored hormone production and the development of folliclescavities in the ovarian tissue where the eggs develop. Within a few months, the monkeys' hormone cycles resumed and they began producing eggs.

The eggs were fertilized through intracytoplasmic sperm injection and the resulting embryos transferred to sur-

gynecology at Weill Cornell Medical College in New York. In March, Otkay and his colleagues reported that they had used the method to restore fertility in a breast cancer survivor (Lancet 363, 837-840; 2004).

Three months after transplantation, the ovarian tissue began to produce hormones and eggs. In the eight months following, Oktay collected 20 eggs, 8 of which looked healthy enough to fertilize in vitro. Of the resulting two embryos, only one was genetically normal. Oktay transferred that embryo to the woman's uterus when it reached the four-cell stage, but the woman did not become pregnant.

Lee and his colleagues have had more sucrogate monkey mothers. Following the normal five-month monkey gestation period, a healthy female infant was born (Nature 428, 137-138; 2004).

The next step would be to try this procedure using frozen ovarian tissue, says Lee.

Most techniques used to safeguard the fertility of cancer patients are relatively new and in need of refinement, but there is a great need for such techniques, says Lynn Westphal, director of Stanford University's oocyte donor program. "Since cancer survivors are doing much better and living longer lives," she says, "this is much more of an issue than it was in the past." Leslie Harris O'Hanlon, Albuquerque 